\title{
Diseño de un módulo de control piloto basado en RFID para retails
}

\begin{abstract}
RESUMEN
El presente artículo analiza el siguiente problema: ¿Qué elementos incluye el diseño de un módulo de control en una empresa comercializadora de prendas de vestir (retail)? Para responder a esta pregunta se procedió a caracterizar un sistema de Identificación por Radio Frecuencia (RFID) aplicable a organizaciones del rubro de comercialización de productos masivos. El estudio se limita a presentar el diseño y los beneficios generales, cualquier análisis económico comparativo o de tasa de retorno dependerá de la empresa en donde se aplique.
\end{abstract}

Palabras clave: Identificación por Radio Frecuencia, control de almacenes, diseño RFID.

\section{DESIGN OF A PILOT CONTROL MODULE BASED IN RFID TO RETAILS ABSTRACT}

This article focused the next problem: What elements does have a design of a module control in a retail?; to answer this question, proceeded to characterizing an identification system by Radio Frequency (RFID) to buying massive products organizations. The research only shows the design and the general benefits, any economic analyze or rate of return will depends of the enterprise where it applies.

Key word: Identification by Radio Frequency, stores control, RFID design.

\section{INTRODUCCIÓN}

El control de productos en un local comercial (retail) es un tema de gran importancia sobre todo para el control de los bienes que dispone la empresa. Una de las tecnologías más usadas es el código de barras, la cual requiere la generación de un código especial que es adherido a un determinado producto.

Para el funcionamiento de un sistema típico de control basado en código de barras, se requiere de una pistola especial de lectura y un operario encargado de realizar la lectura del respectivo código. Sin embargo, este control si bien cumple su cometido, no está del todo automatizado y requiere de una persona dedicada a su funcionamiento.

Una alternativa para este panorama es la Identificación por Radio Frecuencia o RFID por sus siglas en inglés (Radio Frecuency Identification), este sistema representa un adelanto significativo con respecto al código de barras.

El objetivo del presente artículo es mostrar el diseño de una solución RFID aplicada a un retail textil, de tal forma que se pueda ilustrar su aplicación.

\section{TECNOLOGÍA RFID CONCEPTOS GENERALES}

Un sistema RFID se basa en la transmisión de datos por señales de radiofrecuencia; el sistema en general consiste en la colocación de un circuito integrado en el producto a controlar, este chip proporcionará una identificación única a dicho producto; luego mediante una antena y un lector se podrá controlar la ubicación del chip, el cual corresponderá a la ubicación del producto en mención y sus características. Por lo tanto, no es necesario el contacto físico del producto con ningún instrumento de identificación.

La tecnología RFID utiliza actualmente los estándares Frecuencia Ultraalta o UHF (Ultra High Frecuency) lo que le permite lecturas a varios metros; esto supone una mejora con respecto a la tecnología original basada en Alta Frecuencia o HF (High Frecuency), la cual restringía su alcance para distancias cortas.

1 Doctor de Ciencias Administrativas. Profesor del Departamento de Diseño y Tecnología Industrial. UNMSM. E-mail: jlinche@hotmail.com

2 Magíster en Ingeniería Industrial. Profesor del Departamento de Diseño y Tecnología Industrial. UNMSM. E-mail: ramon chung@yahoo.es

3 Ingeniero Industrial. Profesor del Departamento de Producción y Gestión Industrial. UNMSM. E-mail: jasalasb@hotmail.com 


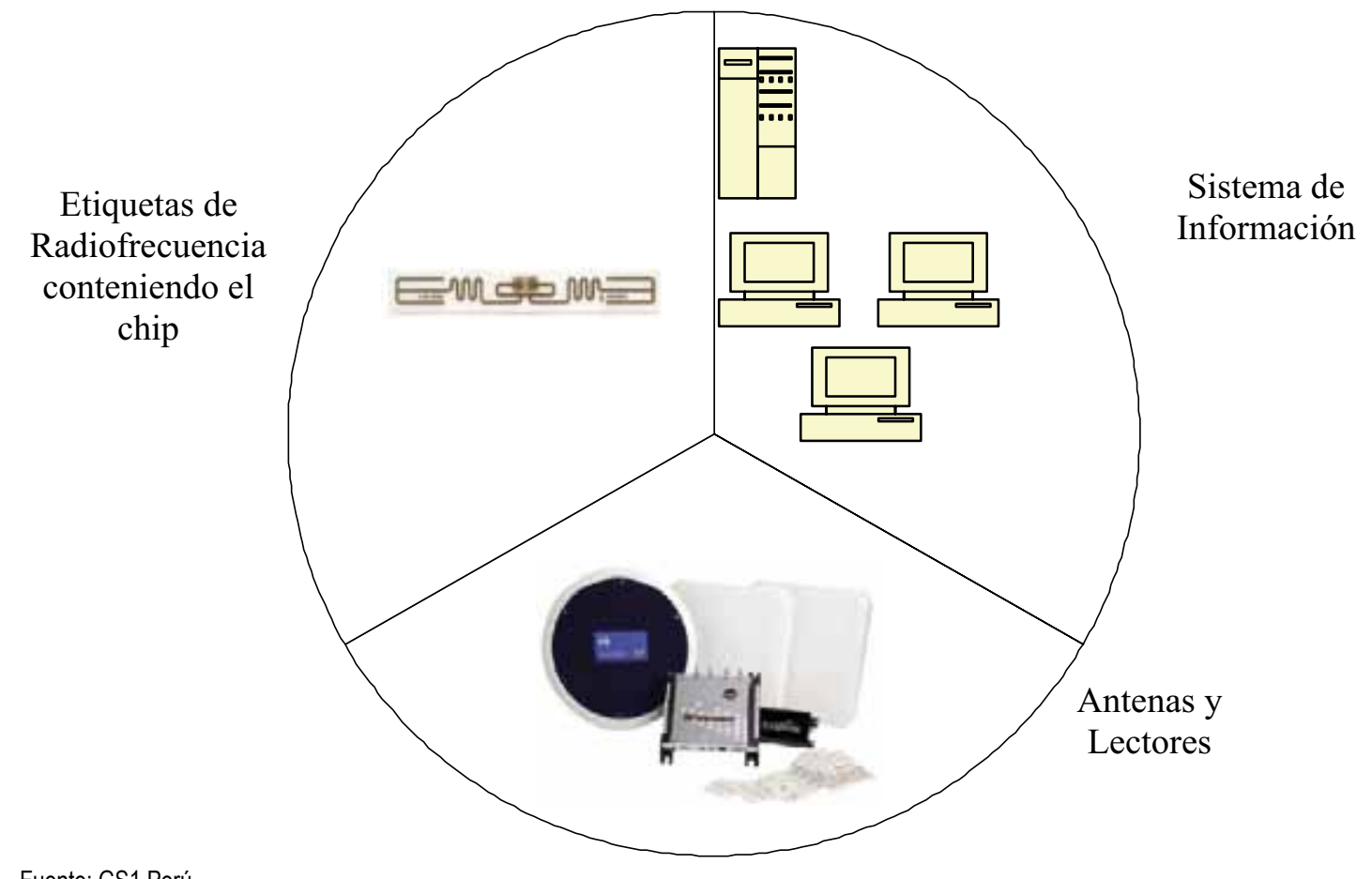

Figura $\mathrm{N}^{\circ}$ 1: Componentes de un sistema de control RFID.

Asimismo es necesario contar con un sistema de información y comunicación que permita la interacción del usuario con el sistema de control de productos en una tienda de prendas de vestir.

El sistema RFID deberá contar con cuatro componentes como mínimo: chip, antena, lector y el sistema de información y comunicación. La Figura N. ${ }^{\circ} 1$ presenta un esquema que ilustra los elementos del sistema de control.
Para la instalación de un sistema RFID se requiere de una estructura que incluye un arco en donde se instalarán las antenas y el lector. La Figura N. ${ }^{\circ} 2$ muestra un sistema de control RFID en donde los paquetes se trasladan a través de un arco.

Entre las ventajas más importantes de un sistema RFID se pueden mencionar:

Mayor seguridad: ya que permite mejorar la trazabilidad y con ello el control y la identificación de productos.

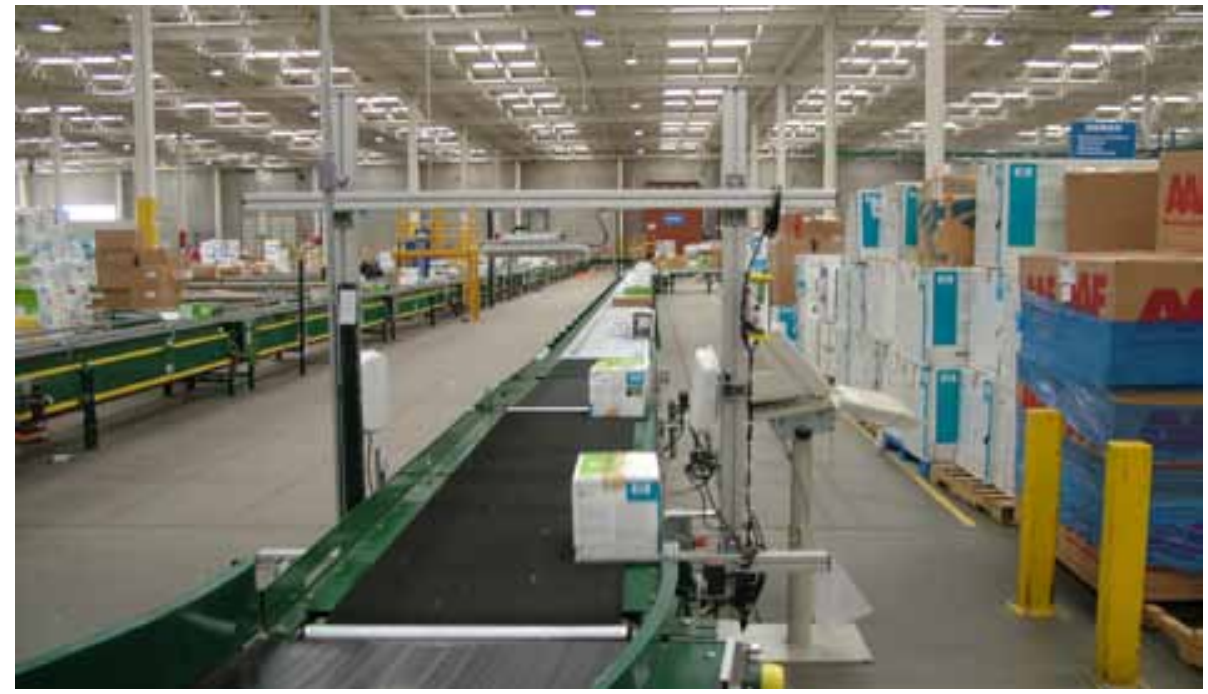

Fuente: GS1 Perú

Figura N. $^{\circ}$ 2: Sistema de control RFID para el control de paquetes. 
Reducción de costos: Ya que el sistema es automatizado, además mejora la calidad del producto y su visibilidad, evitando así distraer recursos y personal en su control.

Por otro lado, con relación al código de barras, el siguiente cuadro ilustra las ventajas de RFID con respecto a esta tecnología:

Cuadro N. ${ }^{\circ}$ 1: Cuadro Comparativo RFID versus Código de Barras.

\begin{tabular}{|l|l|}
\hline Control RFID & Código de barras \\
\hline $\begin{array}{l}\text { Puede identificar en for- } \\
\text { ma individual un produc- } \\
\text { to, es decir con sus ca- } \\
\text { racterísticas propias. }\end{array}$ & $\begin{array}{l}\text { Identifica tipos de } \\
\text { productos con carac- } \\
\text { terísticas generales. }\end{array}$ \\
$\begin{array}{l}\text { No requiere de contacto } \\
\text { visual con el lector, ya } \\
\text { que la lectura se puede } \\
\text { efectuar a una hasta 10 } \\
\text { metros. }\end{array}$ & $\begin{array}{l}\text { Requiere un contacto } \\
\text { visual cercano con el } \\
\text { instrumento lector. }\end{array}$ \\
$\begin{array}{l}\text { Se pueden leer varios } \\
\text { productos en forma pa- } \\
\text { ralela. }\end{array}$ & $\begin{array}{l}\text { La lectura se hace en } \\
\text { forma secuencial. }\end{array}$ \\
$\begin{array}{l}\text { En las etiquetas se pue- } \\
\text { den escribir varias ve- } \\
\text { ces. }\end{array}$ & $\begin{array}{l}\text { Sobre el código de } \\
\text { barras se puede es- } \\
\text { cribir una sola vez. }\end{array}$ \\
\hline
\end{tabular}

Fuente: Ligonio N.,Tecnología RFID en: http://centros5.pntic.mec.es/ies.victoria.kent/Rincon-C/Curiosid2/rc-98/rc-98.htm, con modificaciones de los autores

\section{DISEÑO DE UN SISTEMA RFID PARA UNA EM- PRESA COMERCIALIZADORA DE PRODUCTOS TEXTILES}

Se conoce como retail a las empresas que se dedican al rubro de comercialización de productos masivos; en el caso de estudio, se está considerando empresas del rubro textil.

En este caso, un sistema RFID se debe comenzar desde el etiquetado de la prenda, permitiendo introducir mayor información relacionada a la prenda (por ejemplo color, tipo etc); asimismo debe permitir controlar la ubicación de la prenda. Con estas consideraciones, el proceso es el que se ilustra en el diagrama de bloques de la Figura N. 3.

A continuación se detalla cada componente del diagrama de bloques de la Figura N. ${ }^{\circ} 3$.

\section{Llegadas de prendas al almacén:}

Las prendas llegan al almacén del retail tal y como son remitidas por el proveedor, son recepcionadas según los controles regulares de la empresa.

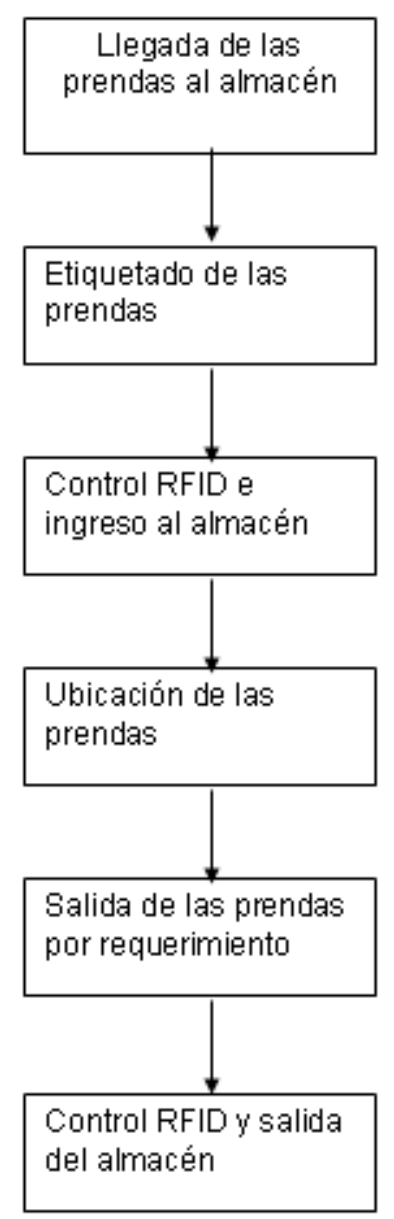

Figura N. ${ }^{\circ}$ 3: Diagrama de bloques del proceso propuesto.

\section{Etiquetado de las prendas:}

Una vez recepcionadas las prendas según el paso anterior, a fin de poder entrar al control RFID se les coloca una etiqueta especial, la cual deberá contener las características necesarias a controlar por cada prenda o tipo de prenda.

\section{Control RFID e ingreso al almacén:}

Como paso siguiente, la prenda pasa mediante una faja transportadora $u$ otro medio de transporte por un arco al cual se le adhieren antenas conectadas a los lectores quienes registran la información de la prenda (específicamente de la etiqueta) hacia el sistema de control RFID.

\section{Ubicación de las prendas:}

Luego de ser registradas las prendas, estas son ubicadas en sus respectivos lugares dentro del almacén hasta la espera de una orden de salida ya sea para muestra o para venta. 


\section{Salida de las prendas por requerimiento:}

Se genera una orden de salida la cual se ejecuta en el almacén, por lo tanto la prenda se prepara para pasar su control de salida.

\section{Control RFID y salida del almacén:}

En forma similar al control de ingreso se efectúa el control de salida, registrando el hecho y los datos necesarios y al mismo tiempo afectando al control de almacén.

\section{IMPLEMENTACIÓN}

El costo de un módulo RFID asciende aproximadamente en S/.32000,00; lo cual incluye 2 lectores, 3 pares de antenas (derecha e izquierda), 1000 etiquetas, licencia, entre otros implementos propios del sistema para su funcionamiento.

La implementación requerida (como todo sistema) requiere de una fase previa de capacitación y adecuación de infraestructura física. Los resultados esperados van desde una mayor rotación del producto, un mejor servicio de ventas, un mejor control de la mercadería, una reducción de stock etc.

\section{CONCLUSIONES}

1. La tecnología RFID presenta mayores ventajas con respecto a la tecnología basada en código de barras.
2. RFID es aplicable a los retails textiles.

3. RFID permite un mayor aprovechamiento del personal asignado al control de inventarios.

4. RFID permite controlar mayor información que una solución basada en código de barras.

\section{REFERENCIAS BIBLIOGRÁFICAS}

[1] ONTSI, AETIC, AT4 (2009). La tecnología RFID: Usos y oportunidades. http://tagingenieros.com/ menu_sup/articulos/RFID_usosOportunidades. pdf. (Visitado el 27-10-2010).

[2] Muñoz J y Camarassa J (2009). Soluciones de Gestión de Almacenes mediante tecnología RFID con aplicación al sector textil - confección. http://tagingenieros.com/menu_sup/articulos/articulo2.php. (Visitado el 27-10-2010).

[3] RFID Magazín (2005). Trazabilidad en la cadena de producción de autobuses. RID Magazín. http://www.rfid-magazine.com/casos/detalle. php?id=150\&class $=($ Visitado el 27-10-2010 $)$.

[4] RFID Journal (2009). Lacoste apuesta por etiquetas RFID para mejorar su logística. RFID Journal. http://www.rfidjournal.com/espanol/casos_de_estudio/vision/4789 (Visitado el 27-102010).

[5] RFID Journal (2009). Farmacias del Ahorro utilizan RFID para control y rastreo de activos. RFID Journal. http://www.rfidjournal.com/espanol/casos_de_estudio/vision/4684 (Visitado el 27-10-2010). 